\title{
Molecular Dynamics of 4-Cyano-3-Fluorophenyl 4-Butylbenzoate as Studied by Dielectric Relaxation Spectroscopy
}

\author{
M. Massalska-Arodź ${ }^{a}$, J. KrawczyK ${ }^{a}$, E. Juszyńska ${ }^{a, b}$, A. Kocot $^{c}$ And A. InabA ${ }^{b}$ \\ ${ }^{a}$ Institute of Nuclear Physics, Polish Academy of Sciences, E. Radzikowskiego 152, 31-342 Kraków, Poland \\ ${ }^{b}$ Research Center for Structural Thermodynamics, Osaka University, Toyonaka, Japan \\ ${ }^{c}$ Institute of Physics, Silesian University, Katowice, Poland
}

\begin{abstract}
Dielectric $\alpha$-relaxation connected with reorientations of the 4-cyano-3-fluorophenyl-4-butylbenzoate molecules around short axes in the isotropic and nematic phases is presented. In the nematic phase the super-Arrhenius temperature dependence of the relaxation has been found. Vitrification of the supercooled nematic phase is shown. Below glass transition temperature the secondary $\beta$-relaxation has been identified and described well by the Arrhenius temperature dependence on cooling and on heating. After softening of glass to the metastable nematic phase a spontaneous crystallization occurs as for some other low-weight molecular glass formers.
\end{abstract}

PACS numbers: 64.70.kj, 64.70.M-, 77.22Gm

\section{Introduction}

Recently, physical properties of materials belonging to the so-called soft matter are in focus of interest due to their complex behavior and a growing number of applications. Such substances, i.e., polymers, colloids, amphiphiles and liquid crystals, show weak transitions between thermodynamic phases, weak interactions of the structural elements, the lack of full long-range ordering of molecules and the complexity of their dynamics [1]. The organic glass formers, i.e. viscous liquids of complex structure of molecules which do not crystallize even on relatively slow cooling but vitrify to form an amorphous solid phase, reveal all those features [2,3]. In the vicinity of glass transition the density of the substance and the local arrangement of the molecules do not change much on lowering temperature, while the viscosity grows dramatically and the diffusional and rotational motions of whole molecules slow down and become frozen [4]. Important is that in supercooled liquid relaxation of fluctuations of density and of molecular configurations etc. to equilibrium is hindered by growth of viscosity. Thus, a non-equilibrium glassy phase of disordered arrangement of spatial and orientational degrees of freedom of molecules is formed.

Liquids of elongated molecules often do not form a glass of isotropic liquid phase but transform on cooling to a liquid crystalline phase which can be supercooled to glassy liquid crystal [5]. In glass transition the anisotropy of the phase does not change and long-range order of some degrees of freedom (a parallel alignment of long molecular axes in nematics or an order of spatial arrangement of molecules in smectics) is frozen-in.
Orientational motions of dipolar molecules allow us to observe the dielectric relaxation process. In given phase the relaxation time $\tau(T)$, describing the rate of molecular reorientations, grows with lowering temperature. The process can be traced by measuring the frequency dependence of the complex electric permittivity $\varepsilon^{*}(\nu)=\varepsilon^{\prime}(\nu)-\mathrm{i} \varepsilon^{\prime \prime}(\nu)$ in the temperature range of interest [6], $\varepsilon^{\prime}(\nu)$ means the dielectric dispersion and $\varepsilon^{\prime \prime}(\nu)$ - the dielectric absorption. In isotropic liquid, on approaching a glass transition temperature $T_{\mathrm{g}}$, the temperature dependence of the dielectric relaxation time $\tau(T)=1 /\left(2 \pi \nu_{\max }(T)\right)$ is non-Arrhenius and fulfils the Vogel-Fulcher-Tammann equation [7, 4]:

$$
\tau(T)=\tau_{0} \exp \left(D T_{0} /\left(T-T_{0}\right)\right),
$$

where $T_{0}$, in general about $20-50 \mathrm{~K}$ below $T_{\mathrm{g}}$ [2], is the temperature of divergence of $\tau(T) . \quad \tau_{0}$ is a relaxation time in the high temperature limit. The magnitude of $1 / D$ can be regarded as a measure of the deviation of the relaxation process observed from the Arrhenius one. It defines the so-called fragility parameter $m$ which is used for classification of glass formers [8]. In the nematic phase the relaxation process has two main contributions connected with reorientations of the molecules around the short axes (relaxation time of about $10^{-6} \mathrm{~s}$ ) and around the long axes (relaxation time of about $10^{-9} \mathrm{~s}$ ) [9]. It is interesting to investigate the temperature dependence of these relaxations on approaching $T_{\mathrm{g}}$. In the paper the results of dielectric measurements of the slower relaxation of 4-cyano-3-fluorophenyl-4-butylbenzoate in the temperature range of the isotropic and nematic phases are presented. Moreover, for temperatures of the glass of nematic phase, the evidence of additional relaxation 
connected with local motions of molecules is given [10]. On heating the process of softening of glass of nematic phase and a crystallization [11] of the supercooled nematic phase and melting of a crystal are shown.

\section{Experimental}

The dielectric measurements of the complex electric permittivity $\varepsilon^{*}(\nu)$ have been performed in the frequency range from $0.03 \mathrm{~Hz}$ to $10 \mathrm{MHz}$ using the Solartron-Schlumberger frequency response analyzer FRA 1260 with the Novocontrol Spectrometer. The resolution of $\varepsilon^{\prime \prime}$ and $\varepsilon^{\prime}$ was smaller than $1 \%$ and $2 \%$, respectively. Measurements cover the temperature region from $130 \mathrm{~K}$ to $300 \mathrm{~K}$. The measurement run for each temperature has started at the higher frequency side and lasted for about 10 min. The sample has been kept between two brass disc-shaped electrodes. The diameter of the cell was $30 \mathrm{~mm}$ while the distance between them was $0.10 \mathrm{~mm}$. Separation between the electrodes was maintained by PTFE spacers. Measurements have been performed at $2 \mathrm{~K}$ intervals on cooling and at $10 \mathrm{~K}$ intervals on heating the sample. On cooling the rate of temperature changes was of about $0.2 \mathrm{~K} / \mathrm{min}$. The temperature of the sample has been controlled using Novocontrol Quatro system with a liquid nitrogen cryostat and measured with the accuracy of $0.1 \mathrm{~K}$. At room temperature the substance is a liquid and differential scanning calorimetry (DSC) measurements show an isotropic-nematic phase transition at $279.9 \mathrm{~K}[12]$

\section{Results and discussion}

\subsection{Cooling}

In Fig. 1 changes of the real $\varepsilon^{\prime}$ and imaginary $\varepsilon^{\prime \prime}$ parts of the electric permittivity observed on cooling of the isotropic I and nematic $\mathrm{N}$ phases are presented vs. $\log \nu$. The relaxation curves observed in the isotropic phase at the $\mathrm{MHz}$ frequency range are connected with reorientations of the effective dipolar moment $\mu$ of the molecule around short axes. In 4-cyano-3-fluorophenyl-4-butylbenzoate molecule

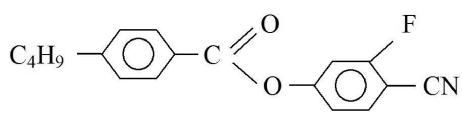

three molecular groups $(\mathrm{CO}, \mathrm{CF}$ and $\mathrm{CN})$ contribute to the dipolar moment $\mu$.

At about $280 \mathrm{~K}$ a transformation of the $\varepsilon^{\prime \prime}(\log \nu)$ curves was observed due to the $\mathrm{I} \rightarrow \mathrm{N}$ phase transition. In the nematic phase, alignment of the long molecular axes causes essential growth of the effective dipolar moment of reorienting molecules perpendicular to the long axes observed as a jump of the dielectric increment $\Delta \varepsilon$ to about $30\left(\varepsilon_{\max }^{\prime \prime} \approx 12.5\right)$ from about 14 in the isotropic phase $\left(\varepsilon_{\max }^{\prime \prime} \approx 7\right)$. At the phase transition a small

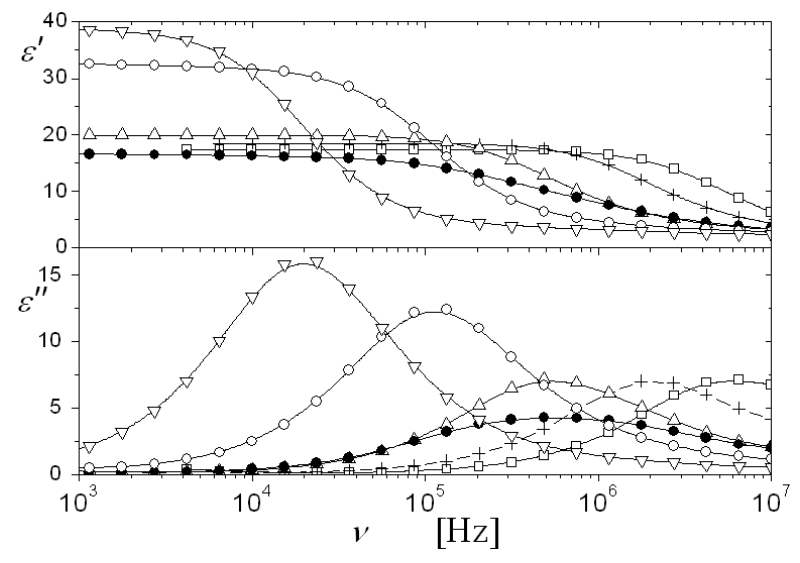

Fig. 1. Frequency dependence of the real $\varepsilon^{\prime}$ and imaginary $\varepsilon^{\prime \prime}$ parts of the electric permittivity of the 4-cyano-3-fluorophenyl-4-butylbenzoate observed on cooling at several temperatures of the isotropic (303 K $(\square)$, $293 \mathrm{~K}(+)$ and $281 \mathrm{~K}(\triangle)$ ) and nematic (at $277 \mathrm{~K}(\mathrm{o})$ and $267 \mathrm{~K}(\nabla))$ phases. Data for $279 \mathrm{~K}(\bullet)$ measured during phase transition.

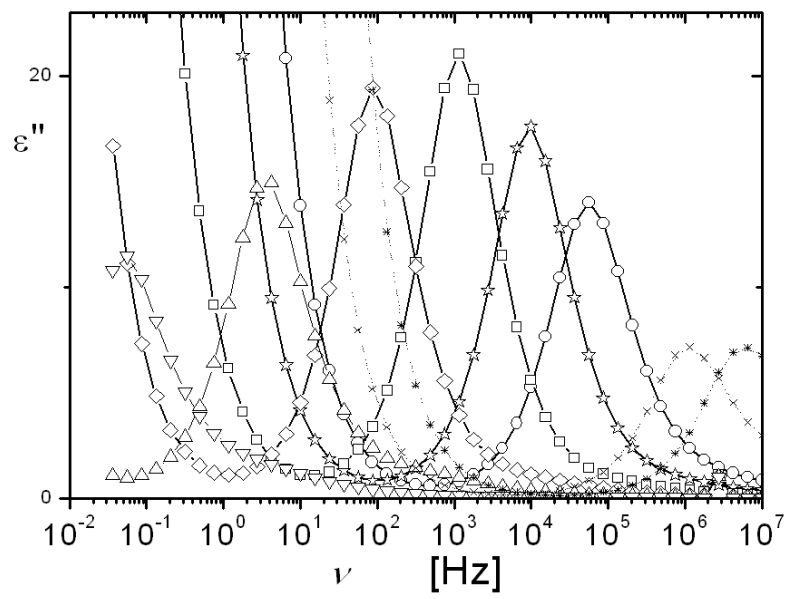

Fig. 2. Absorption $\varepsilon^{\prime \prime}(\log \nu)$ presented for several temperatures on cooling. Meaning of the symbols is the following: $*-303 \mathrm{~K}, \times-283 \mathrm{~K}, \circ-273 \mathrm{~K}$, $\star-263 \mathrm{~K}, \quad \square-253 \mathrm{~K}, \diamond-243 \mathrm{~K}, \Delta-233 \mathrm{~K}$, $\nabla-223 \mathrm{~K}$.

drop of the rate of molecular reorientations was also detected. On cooling the nematic phase no crystallization was detected.

Shift of the absorption curves $\varepsilon^{\prime \prime}(\log \nu)$ presented in Fig. 2 on lowering temperature each $10 \mathrm{~K}$ shows a slowing down of the relaxation due to supercooling and then vitrification of the nematic phase. The relaxation time $\tau=100 \mathrm{~s}$ was detected at the temperature $T_{\mathrm{g}} \approx 220 \mathrm{~K}$, regarded as the glass transition temperature in dielectric experiments $[2,3,9]$.

It was found that an extra very weak absorption of $\varepsilon_{\max }^{\prime \prime}<0.05$ is a component of main absorption at low temperatures as shown in Fig. 3. The process survives a 


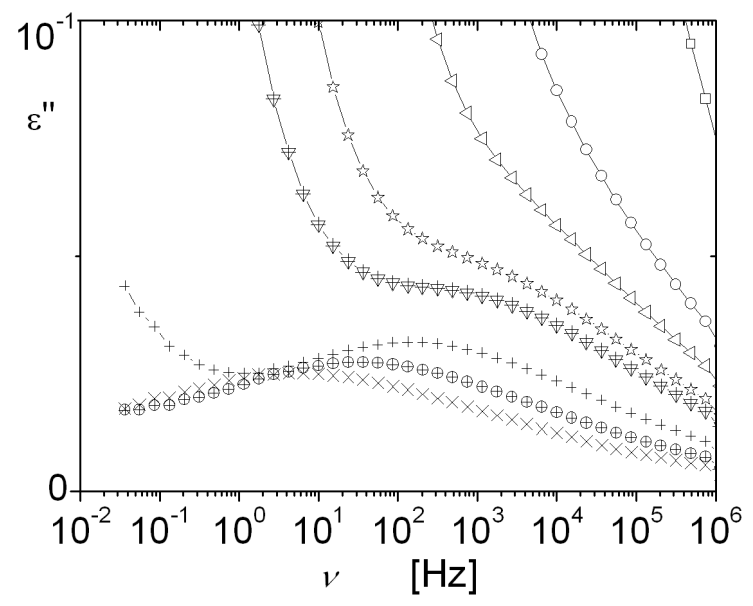

Fig. 3. Absorption $\varepsilon^{\prime \prime}(\log \nu)$ related to the $\beta$ relaxation presented on cooling at low temperature region. Meaning of the symbols is the following: $\square-227 \mathrm{~K}, \quad \circ-223 \mathrm{~K}, \quad \triangleleft-219 \mathrm{~K}, \quad \star-215 \mathrm{~K}$, $\forall-213 \mathrm{~K},+-203 \mathrm{~K}, \oplus-193 \mathrm{~K}, \times-183 \mathrm{~K}$.

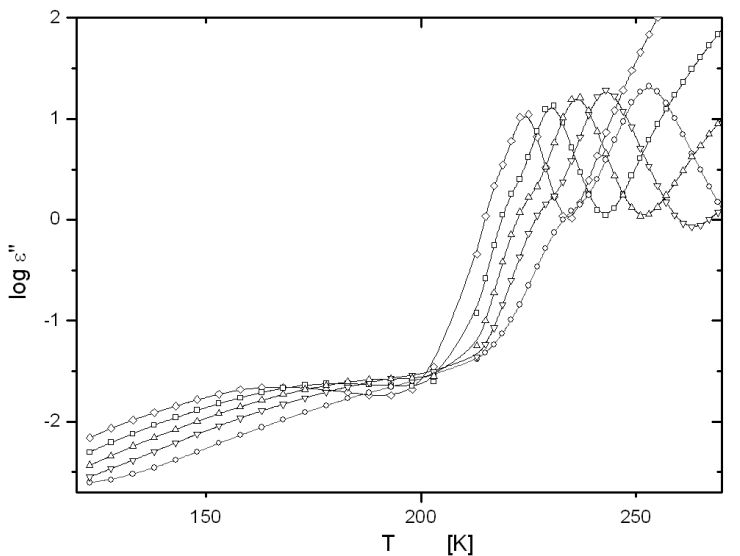

Fig. 4. Temperature dependence of the absorption $\log \varepsilon^{\prime \prime}(T)$ showing $\beta$-relaxation and $\alpha$-relaxation at the following frequencies: $\diamond-0.1 \mathrm{~Hz}, \square-1 \mathrm{~Hz}$, $\triangle-10 \mathrm{~Hz}, \nabla-100 \mathrm{~Hz}, \circ-1000 \mathrm{~Hz}$.

glass transition. Below $200 \mathrm{~K}$, it is the only relaxation detected in the frequency range available.

In Fig. 4 the temperature dependence of the $\log \varepsilon^{\prime \prime}$ is presented for several frequencies used. One can see two mentioned processes: the main structural $\alpha$-relaxation above $T_{\mathrm{g}}$ and a secondary $\beta$-relaxation [10]. Moreover, it is visible that the main process is not of the Debye shape and has a shoulder on the lower temperature side related to the faster relaxation.

The absorption curves $\varepsilon^{\prime \prime}(\nu)$ of the $\alpha$-relaxation were separated into two components in the best way using the Cole-Davidson formula (see Fig. 5) [6]:

$$
\begin{aligned}
& \varepsilon^{*}(\nu)=\varepsilon_{\infty}+\Delta \varepsilon_{1} /\left(1+\mathrm{i} \omega \tau_{1}\right)^{\beta_{1}}+\Delta \varepsilon_{2} /\left(1+\mathrm{i} \omega \tau_{2}\right)^{\beta_{2}} \\
& \quad+A /\left(\nu-\nu_{0}\right) .
\end{aligned}
$$

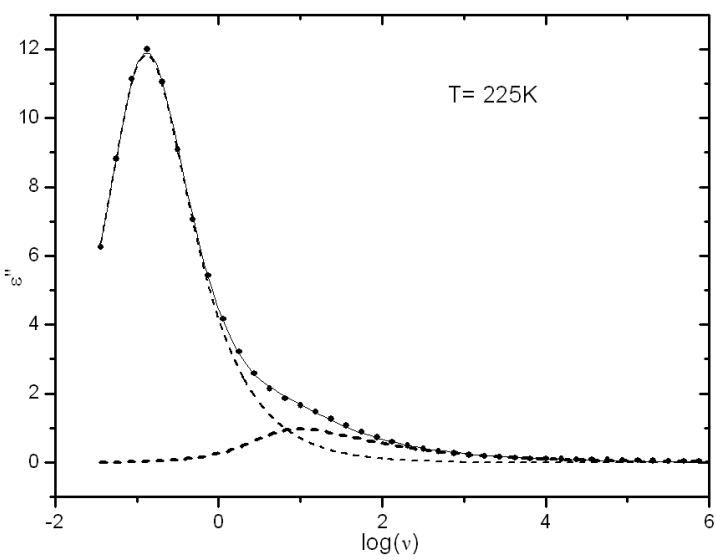

Fig. 5. Separation of the absorption of the $\alpha$-relaxation into two processes by fitting two Cole-Davidson formulae for the data at $225 \mathrm{~K}$.

The last term describes the electric conductivity observed at high temperatures. The exponents $0<\beta_{1}, \beta_{2} \leq 1$ describe the distributions of the relaxation times $\tau_{1}$ and $\tau_{2}$. The value $\beta=1$ corresponds to a single Debye process. For $225 \mathrm{~K}$ the fitting parameters are the following: $\Delta \varepsilon_{1}=28.9, \Delta \varepsilon_{2}=2.94, \tau_{1}=9.89 \mathrm{~s}, \tau_{2}=0.18 \mathrm{~s}$, $\beta_{1}=0.75$, and $\beta_{2}=0.38$. Estimated error of the parameters does not exceed $10 \%$.

For the description of the $\beta$-relaxation, presented in Fig. 3 below $223 \mathrm{~K}$, the Havriliak-Negami formula [13] was used

$$
\varepsilon^{*}(\nu)=\varepsilon_{\infty}+\Delta \varepsilon /\left[1+(\mathrm{i} \omega \tau)^{1-\alpha}\right)^{\beta}+A /\left(\nu-\nu_{0}\right],
$$
with $0<(1-\alpha), \beta(1-\alpha) \leq 1$. For $\beta(1-\alpha)=(1-\alpha)=1$ the process is of the Debye type. For $188 \mathrm{~K}$ the following values of the parameters were obtained in the fitting procedure: $(1-\alpha) \sim \beta(1-\alpha) \approx 0.24, \Delta \varepsilon=0.27$ and $\tau=0.058 \mathrm{~s}$. The absorption curves of the $\beta$-relaxation are symmetrical as illustrated by $(1-\alpha) \sim \beta(1-\alpha)$. The deviation of the $\beta$-relaxation from the Debye shape is large. One can see that the half-maximum width $W=5.58$ of the $\beta$ absorption peak exceeds significantly $W_{\mathrm{D}}=1.14$, the width of the Debye absorption curve in the logarithmic scale. In the Kolhrausch-WilliamsWatts stretched exponential relaxation function [14], the exponent $W_{\mathrm{D}} / W=0.17$ determines the wide shape of the $\beta$-relaxation and distribution of the relaxation times. Temperature dependence of shape exponents for two $\alpha$-relaxations and the $\beta$-relaxation is presented in Fig. 6 .

In Fig. 7 the dependence of the $\log \tau$ vs. $1000 / T$ is presented for two components contributing to the $\alpha$-relaxation and for the $\beta$-relaxation. In the nematic phase the structural relaxation is shown by circles (the main process) and triangles (higher frequency component). The temperature dependence of dynamics related to the structural relaxation is of the super-Arrhenius type with the following parameters obtained by fitting Eq. (1) to the data: $T_{0}=150 \mathrm{~K}$ and $D=16$ for the main process and $D=14.4$ for the weaker process, $\tau_{0}$ was 


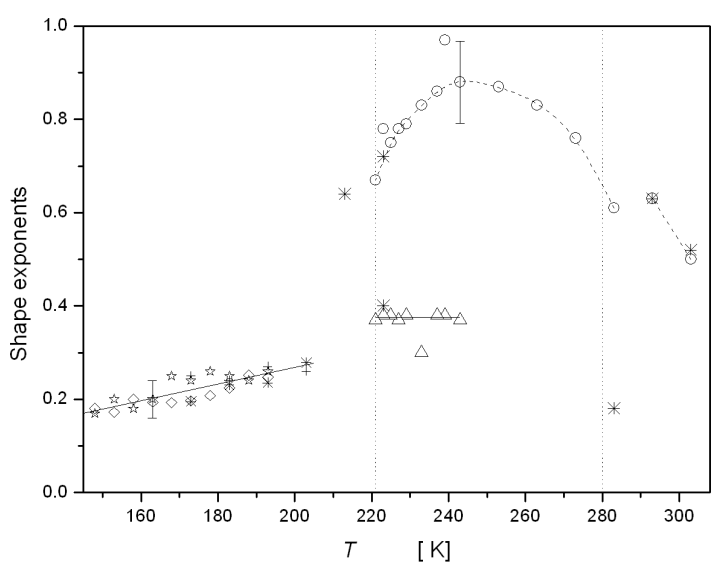

Fig. 6. The temperature changes of the shape exponents. Dotted lines mark the temperature of the isotropic-nematic phase transition and glass transition temperature. Open symbols are for data observed on cooling: $\circ-$ for $\beta_{1}$ of the main $\alpha$-relaxation, $\Delta-$ for $\beta_{2}$ of the higher frequency $\alpha$-relaxation while $\diamond-$ for parameter $(1-\alpha)$ and $\star-$ for parameter $\beta(1-\alpha)$ of the $\beta$-relaxation. Symbols $*$ and + are for the data obtained on heating. In the range from $233 \mathrm{~K}$ to $273 \mathrm{~K}$ no data on heating are shown due to spontaneous crystallization.

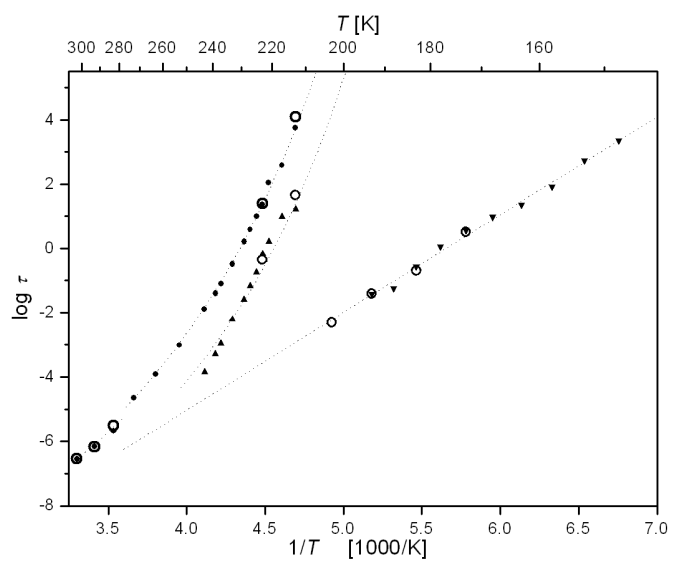

Fig. 7. Temperature changes of the relaxation times $\log \tau(1 / T)$ for two $\alpha$-relaxation processes and for the $\beta$ relaxation process. Meaning of the symbols for cooling is the following: - - for the main and $\boldsymbol{\Delta}-$ for the higher frequency $\alpha$-relaxations, $\boldsymbol{\nabla}$ - for $\beta$-relaxation. The points marked by $\circ-$ are for heating. Dotted lines are fitted using the Vogel-Fulcher-Tammann formula and the Arrhenius formula to the data obtained on cooling.

fixed as $10^{-13.5} \mathrm{~s}$. The secondary process is of the Arrhenius type with the activation enthalpy of $14.72 \mathrm{kcal} / \mathrm{mole}$ $(61.67 \mathrm{~kJ} / \mathrm{mole})$. In the isotropic phase only a single process was observed.

\subsection{Heating}

Heating of the substance was started at $130 \mathrm{~K}$, i.e., in the glass of nematic phase. The absorption curves ob- served are presented in Fig. 8. As on cooling, only the $\beta$-process was detected up to $200 \mathrm{~K}$. One can see that the parameters (shape exponents and relaxation time) of the $\beta$-relaxation on heating, marked by $*$ in Fig. 6 and by $\circ$ in Fig. 7, are the same as on cooling. Above $220 \mathrm{~K}$, after softening of the glass to the metastable nematic phase, the $\alpha$-process appeared in the used frequency range. At $233 \mathrm{~K}$ a spontaneous crystallization [11] started to occur causing a decrease of the absorption (compare $\circ$ in Fig. 8 with $\Delta$ in Fig. 2, on cooling) due to diminishing of the number of reorienting molecular dipoles. At that temperature the substance is no longer in equilibrium so description of the relaxation by formula (2) was not possible. Above $233 \mathrm{~K}$ dynamics of molecules was stopped and no structural relaxation was visible till $283 \mathrm{~K}$ when regions of liquid phase start to grow in the sample.

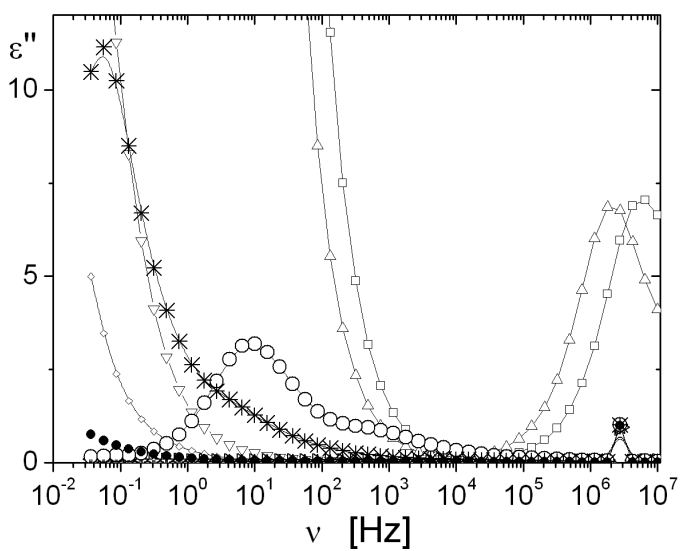

Fig. 8. Absorption curves on heating. Meaning of the symbols in glass of nematic phase: - - $203 \mathrm{~K}$, $\diamond-213 \mathrm{~K}$, in the supercooled nematic: $*-223 \mathrm{~K}$, during a spontaneous crystallization: $\circ-233 \mathrm{~K}$, in the crystal: $\nabla-243 \mathrm{~K}$ (only electric conductivity contribution measured) and in the isotropic phase: $\triangle-283 \mathrm{~K}, \square-293 \mathrm{~K}$.

One can see that the * point calculated for $283 \mathrm{~K}$ (Fig. 6) differs much from the corresponding one on cooling which shows that melting is still not finished. In the adiabatic calorimetry measurements [15] the maximum of the anomaly in heat capacity vs. $T$ observed on heating at $288.4 \mathrm{~K}$ was identified as the melting of one of the crystalline phases found (crystal I). Careful analysis of dielectric data in the temperature range between 243 and $263 \mathrm{~K}$ allowed to find a trace of slow, very weak absorption with $\varepsilon_{\max }^{\prime \prime}$ smaller than $0.01 \mathrm{~K}$ and $\tau \approx 0.01 \mathrm{~s}$ at $253 \mathrm{~K}$. This relaxation can be related to some conformational dynamics in the lower temperature crystalline phase (crystal II) which transforms at $260 \mathrm{~K}$ to the crystal I as detected by the adiabatic calorimetry [15].

\section{Conclusions}

The 4-cyano-3-fluorophenyl-4-butylbenzoate occurs to be a glass former. The substance forms a monotropic 
system of phases, namely two stable phases: crystal I $288.4 \mathrm{~K}$ - isotropic phase, and three metastable phases: glass of the nematic phase - $220 \mathrm{~K}$ - nematic phase $233 \mathrm{~K}$ - crystal II. A spontaneous crystallization occurs on heating at $233 \mathrm{~K}$, as in several low weight molecular compounds. In the isotropic phase the $\mathrm{MHz}$ dielectric relaxation was measured related to the motions of the molecules around short axes. Below $280 \mathrm{~K}$, the temperature of the isotropic to nematic phase transition, the $\alpha$-relaxation was identified. It was resolved using two Cole-Davidson formulae into main process related to the reorientations of molecules around short axes and much smaller component which could be related to the faster precession of the molecules [16]. Both processes exhibit the super-Arrhenius temperature dependence with the same Kauzmann temperature $T_{0}=150 \mathrm{~K}$. Below glass transition the secondary $\beta$-relaxation of the Arrhenius type was detected and described by the HavriliakNegami formula. Dielectric measurements under elevated pressure are planned for better understanding of the mechanism of the relaxations observed.

\section{Acknowledgments}

The authors would like to thank Professor Roman Dąbrowski (Military University of Technology, Warszawa) for preparation of the substance.

\section{References}

[1] I.W. Hamley Introduction to the Soft Matter, Wiley 2007
[2] C.A. Angell, Science 267, 1924 (1996).

[3] E. Donth, Glass Transitions, Springer-Verlag, Berlin 2001.

[4] M. Cohen, G.S. Grest, Phys. Rev. B 20, 1077 (1979).

[5] M. Sorai, S. Seki, Mol. Cryst. Liq. Cryst. 19, 299 (1973).

[6] C. Böttcher, P. Bordewijk, Theory of Electric Polarization, Vol. II, Elsevier Sci., Amsterdam 1978.

[7] G. Tammann, G. Hesse, Z. Anorg. Allg. Chem. 156, 245 (1926).

[8] R. Böhmer, K.L. Ngai, C.A. Angell, D. Plazek, J. Chem. Phys. 99, 4201 (1993).

[9] H. Kresse, Adv. Liq. Cryst. 6, 109 (1983).

[10] G.P. Johari, M. Goldstein, J. Chem. Phys. 53, 2372 (1970).

[11] M. Massalska-Arodź, G. Williams, I.K. Smith, Ch. Connoly, R. Dąbrowski, J. Chem. Soc. Faraday Trans. 94, 387 (1998).

[12] R. Dąbrowski, unpublished results.

[13] S. Havriliak, S. Negami, Polymer 8, 161 (1967).

[14] G. Williams, D.C. Watts, Trans. Faraday Soc. 66, 80 (1970).

[15] A. Inaba, unpublished results.

[16] M. Massalska-Arodź, J. Mościcki, S. Wróbel, Acta Phys. Pol. A 58, 443 (1980). 